\title{
The Correction of Fiber Throughput Variation due to Focal Ratio Degradation
}

\author{
Jianjun Chen, Zhongrui Bai, Guangwei Li and Haotong Zhang \\ National Astronomical Observatories, Chinese Academy of Sciences 20A Datun Road, \\ Chaoyang District, Beijing, China \\ email: jjchen@nao.cas.cn
}

\begin{abstract}
The focal ratio degradation (FRD) of optical fibers is a major source causing light loss to astronomical multi-fibre instruments like LAMOST (Oliveira, A. C, et al. 2005). The effects of stress and twist during mounting and rotation of the fibers could change the FRD for individual fibers (Clayton 1989), which means that the transmission efficiency of each individual fiber will vary. We investigate such throughput variation among LAMOST fibers and its relevance to the intensity of sky emission lines (Garstang 1989) over the full wavelength coverage. On the basis of the work, we present an approach to correct the varied fiber throughput by measuring the strength of the sky emission lines as the secondary throughput correction.
\end{abstract}

Keywords. fiber throughput variation, focal ratio degradation

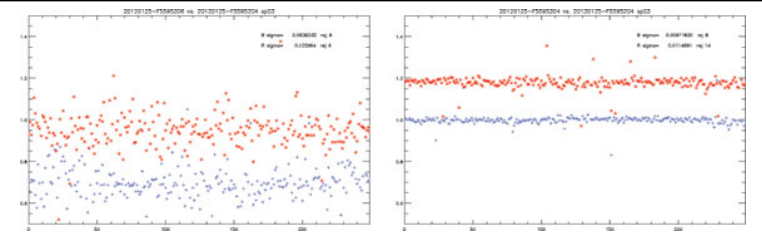

Figure 1. Sky emission lines can be taken as indicators of fiber throughput. Left: Ratio of $\lambda \lambda 5577$ (blue) and $\lambda \lambda 8919$ (red) between two consecutive exposures, but with two different fiber positioning configurations, i.e., fibers rotate between two exposures. Right: Ratio of $\lambda \lambda 5577$ (blue) and $\lambda \lambda 8919$ (red) between two consecutive exposures, sharing same fiber positioning configuration, i.e., no rotation between two exposures.
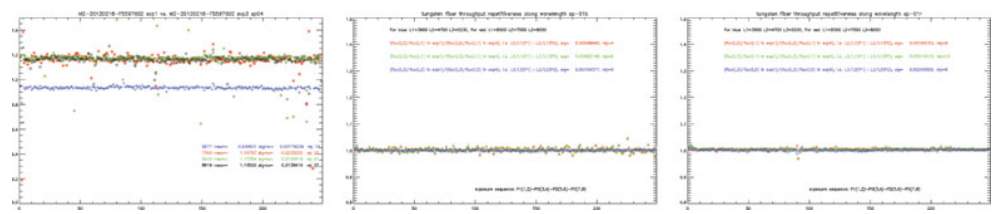

Figure 2. Fiber throughput varies in the same way along all wavelengths. Left: ratio of multiple sky emission lines between two exposures with different fiber positioning configurations. Middle and Right: Ratio of two dome flat fielding exposures with tungsten lamp as light source, having different fiber positioning configurations in blue(middle) and red(right) branch.

\section{References}

Clayton, C. A. $1989, A A, 213,502$

Oliveira, A. C, et al. 2005, MNRAS, 356, 1079

Garstang, R. H. 1989, PASP, 101, 306 